\title{
Accounting for adsorption and desorption in lattice Boltzmann simulations
}

\author{
Maximilien Levesque, ${ }^{1,2,3, *}$ Magali Duvail,,${ }^{1,2,4}$ Ignacio Pagonabarraga, ${ }^{5}$ Daan Frenkel, ${ }^{6}$ and Benjamin Rotenberg ${ }^{1,2}$ \\ ${ }^{1}$ UPMC Univ Paris 06, UMR 7195, PECSA, F-75005, Paris, France \\ ${ }^{2}$ CNRS, UMR 7195, PECSA, F-75005, Paris, France \\ ${ }^{3}$ Department of Materials, University of Oxford, Parks Road, Oxford OX1 3PH, United Kingdom \\ ${ }^{4}$ Institut de Chimie Séparative de Marcoule, UMR 5257, CEA-CNRS-Université Montpellier 2-ENSCM, Site de Marcoule, \\ BP 17171, F-30207 Bagnols-sur-Cèze, France \\ ${ }^{5}$ Departament de Física Fonamental, Universitat de Barcelona, 08028 Barcelona, Spain \\ ${ }^{6}$ Department of Chemistry, University of Cambridge, Lensfield Road, Cambridge CB2 1EW, United Kingdom
}

(Received 29 January 2013; published 19 July 2013)

\begin{abstract}
We report a Lattice-Boltzmann scheme that accounts for adsorption and desorption in the calculation of mesoscale dynamical properties of tracers in media of arbitrary complexity. Lattice Boltzmann simulations made it possible to solve numerically the coupled Navier-Stokes equations of fluid dynamics and Nernst-Planck equations of electrokinetics in complex, heterogeneous media. With the moment propagation scheme, it became possible to extract the effective diffusion and dispersion coefficients of tracers, or solutes, of any charge, e.g., in porous media. Nevertheless, the dynamical properties of tracers depend on the tracer-surface affinity, which is not purely electrostatic and also includes a species-specific contribution. In order to capture this important feature, we introduce specific adsorption and desorption processes in a lattice Boltzmann scheme through a modified moment propagation algorithm, in which tracers may adsorb and desorb from surfaces through kinetic reaction rates. The method is validated on exact results for pure diffusion and diffusion-advection in Poiseuille flows in a simple geometry. We finally illustrate the importance of taking such processes into account in the time-dependent diffusion coefficient in a more complex porous medium.
\end{abstract}

DOI: 10.1103/PhysRevE.88.013308

PACS number(s): 47.11.-j, 47.55.dr, 47.56.+r, 47.61.-k

\section{INTRODUCTION}

The dynamical properties of fluids in heterogeneous materials offer a great challenge and have implications in many technological and environmental contexts. Inherently multiscale in time and space, mesoscale properties such as the diffusion or dispersion coefficient reflect the nanoto microscopic geometry of the media and the interatomic interactions between flowing particles, or tracers, and surface atoms. Experimentally, information about the microstructure of porous media can be extracted from diffusion measurements by pulsed gradient spin echo nuclear magnetic resonance (PGSE-NMR) [1-3]. At short times, the dynamics of a pulse of tracers is connected to the geometry of the porous medium at the pore scale. At longer times, macroscale properties such as porosity and tortuosity come into play. Theoretically, stochastic approaches have been an important support to the understanding of the underlying phenomena [2-4] and have been used recently to show that adsorption and desorption processes may strongly modify the short and long time dynamics of the tracers $[5,6]$.

In numerous, if not all, practical situations involving particle diffusion and advection, the carrier fluid is in contact with confining walls where adsorption may occur. These processes depend on the chemical nature of the solute, which explains why particles with the same charge may diffuse in the same medium with different effective diffusion coefficients. This species-dependent affinity is at the heart of all chromatographic techniques used in analytic and separation chemistry [7]. It also plays a crucial role in the dissemination

\footnotetext{
*maximilien.levesque@gmail.com
}

of toxic or radioactive pollutants in the environment and, conversely, in remediation strategies. Recently, the great interest in nanofluidic devices and in the transport in heterogeneous porous media has also raised the issue of the relevance of models which do not take into account these sorption processes. Moreover, it was recently shown that stochastic resonance between these processes and some external field may be of practical importance, e.g., for molecular sorting $[5,8]$.

At the mesoscale, the dynamics of particles in a fluid can be described by the continuity equation

$$
\partial_{t} \rho(\mathbf{r}, t)=-\nabla \cdot \mathbf{J}(\mathbf{r}, t)
$$

where $\rho(\mathbf{r}, t)$ is the one-particle density at position $\mathbf{r}$ and time $t$, $\partial_{t} \equiv \partial / \partial t$, and $\mathbf{J}$ is the particle flux, which is a function of the velocity field of the carrier fluid, the bulk diffusion coefficient $D_{b}$ of the particles, and, if any, their charge and the local electric field arising from their environment. If adsorption is taken into account, solid-liquid interfaces located at $\mathbf{r}$ have a surface concentration $\Gamma(\mathbf{r}, t)\left(\right.$ length $\left.^{-2}\right)$ that evolves with time according to

$$
\partial_{t} \Gamma(\mathbf{r}, t)=-k_{d} \Gamma(\mathbf{r}, t)+k_{a} \rho(\mathbf{r}, t),
$$

where $k_{a}$ (length time ${ }^{-1}$ ) and $k_{d}\left(\right.$ time $^{-1}$ ) are kinetic adsorption and desorption rates. For molecules, the rates can vary widely. As an example, the dissociation rate of DNA double strands on a surface grafted with single-strand DNA ranges from $10^{-5}$ to $10^{-3} \mathrm{~s}^{-1}$ for a few tens of base pairs [9]. Their adsorption rate can be adjusted by changing the grafting density. Finally, we assume that the tracers (the solutes) neither diffuse into the solid phase (even though that process can easily be accounted for using our algorithm) nor dissolve the surfaces [10]. 
The time-dependent diffusion coefficient $D(t)$ and the dispersion coefficient $K$ of the tracers can be investigated by following the spreading of a tracer pulse in the fluid. This would amount to solving Eqs. (1) and (2), e.g., with a finite element method, for all possible initial conditions, which is computationally intractable for complex systems such as heterogeneous porous media. An alternative is to deduce $K$ and $D(t)$ from the tracer velocity autocorrelation function (VACF) following [11-14]

$$
\begin{gathered}
D_{\gamma}(t)=\int_{0}^{t} Z_{\gamma}\left(t^{\prime}\right) d t^{\prime}, \\
K_{\gamma}=\int_{0}^{\infty}\left[Z_{\gamma}(t)-Z_{\gamma}(\infty)\right] d t,
\end{gathered}
$$

where the VACF in the direction $\gamma \in\{x, y, z\}$ is

$$
Z_{\gamma}(t)=\left\langle v_{\gamma}(0) v_{\gamma}(t)\right\rangle \text {. }
$$

At long times $Z_{\gamma}(\infty)=\bar{v}_{\gamma}^{2}$, with $\bar{v}_{\gamma}$ being the average velocity of the flow. The issue of averaging over initial conditions in Eq. (5) can be handled elegantly and efficiently using the moment propagation method $[12,15,16]$, which was recently extended to charged tracers [14,17-21].

In order to compute the VACF of the tracers from Eq. (5), one has to keep track of their velocity. For this purpose, we use the underlying dynamics of the fluid given by Eq. (1), which does not rely on the velocity of individual particles but on the one-particle solvent density. Moreover, the simulation of heterogeneous multiscale media requires a numerically efficient method. The lattice Boltzmann (LB) method [22-28] offers a convenient framework to deal with such situations. In the LB approach, the fundamental quantity is a one-particle velocity distribution function $f_{i}(\mathbf{r}, t)$ that describes the density of particles with velocity $\mathbf{c}_{i}$, typically discretized over 19 values for three-dimensional LB, at a node $\mathbf{r}$, either fluid or solid, of a lattice of spacing $\Delta x$ and at a time $t$ discretized by steps of $\Delta t$. The dynamics of the fluid are governed by transition probabilities of a particle moving in the fluid from one node to the neighboring ones:

$$
f_{i}\left(\mathbf{r}+\mathbf{c}_{i} \Delta t, t+\Delta t\right)=f_{i}(\mathbf{r}, t)+\Delta_{i}(\mathbf{r}, t),
$$

where $\Delta_{i}$, the so-called collision operator, is the change in $f_{i}$ due to collisions at lattice nodes. This LB equation recovers the fluid dynamics of a liquid, and the moments of the distribution function are related to the relevant hydrodynamic variables. The reader is referred to Refs. [26] and [28] for reviews of the method.

\section{ALGORITHM}

In order to compute the dynamical properties of tracers evolving in a fluid described by the LB algorithm, i.e., to solve Eqs. (3)-(5), we use the moment propagation (MP) method [11,12]. Other methods could have been used, such as the numerical resolution of the macroscopic equations or Brownian dynamics to simulate the random walk of tracers biased by the LB flow [29]. The latter method has often been successfully used, e.g., by Boek and Venturoli [30]. Nevertheless, the MP method offers many advantages. First, MP relies on the same ground as LB. It is based on the propagation of a position and velocity distribution function $[12,15]$, therefore offering an elegant unified approach. Second, MP allows for the propagation of any moment of the distribution function $f_{i}(\mathbf{r}, t)$, which offers great opportunities. For example, Lowe et al. exploited these higher moments to compute self-dynamic structure factors [31]. The LB-MP method has been thoroughly validated by Merks for low Péclet and Reynolds numbers [16].

In the moment propagation algorithm, any quantity $P(\mathbf{r}, t)$ can be propagated between fluid nodes. This quantity will be modified by adsorption and desorption processes. In their absence, $P(\mathbf{r}, t+\Delta t)=P^{\star}(\mathbf{r}, t+\Delta t)$, with

$$
\begin{aligned}
P^{\star}(\mathbf{r}, t+\Delta t)= & \sum_{i}\left[P\left(\mathbf{r}-\mathbf{c}_{i} \Delta t, t\right) p_{i}\left(\mathbf{r}-\mathbf{c}_{i} \Delta t, t\right)\right] \\
& +P(\mathbf{r}, t)\left(1-\sum_{i} p_{i}(\mathbf{r}, t)\right)
\end{aligned}
$$

where the first sum runs over all discrete velocities connecting adjacent nodes. The probability of leaving node $\mathbf{r}$ along the direction $\mathbf{c}_{i}$ is denoted $p_{i}(\mathbf{r}, t)$. The last term in Eq. (7) represents the fraction of particles that did not move from $\mathbf{r}$ at the previous time step. The expression for $p_{i}$, which is central in the algorithm, depends on the nature of the tracers. It is given by

$$
p_{i}(\mathbf{r}, t)=\frac{f_{i}(\mathbf{r}, t)}{\rho(\mathbf{r}, t)}-\omega_{i}+\frac{\omega_{i} \lambda}{2} Q,
$$

where the first two terms account for advection and are obtained by coupling the tracer dynamics to that of the fluid evolving according to the LB scheme. The weights $\omega_{i}$ are constants depending upon the underlying LB lattice. The last term describes diffusive mass transfers. The dimensionless parameter $\lambda$ determines the bulk diffusion coefficient $D_{b}=$ $\lambda c_{s}^{2} \Delta t / 4$, with $c_{s}=\sqrt{k_{B} T / m}$ being the sound velocity in the fluid. It also determines the mobility of tracers under the influence of chemical potential gradients (including the electrostatic contribution), which are accounted for in the $Q$ term as described in Ref. [17]. For neutral tracers, $Q=1$.

We now introduce a propagation scheme to account for adsorption and desorption at the solid-liquid interface. While Eq. (7) still holds for nodes $\mathbf{r}$ which are in the fluid but not at the interface, for the fluid interfacial nodes we define a propagated quantity $P_{\text {ads }}(\mathbf{r}, t)$ associated with adsorbed particles:

$$
P_{\mathrm{ads}}(\mathbf{r}, t+\Delta t)=P(\mathbf{r}, t) p_{a}+P_{\mathrm{ads}}(\mathbf{r}, t)\left(1-p_{d}\right),
$$

where $p_{a}=k_{a} \Delta t / \Delta x$ is the probability for a tracer lying at an interface to adsorb and $p_{d}=k_{d} \Delta t$ is the probability for an adsorbed, immobile tracer to desorb. There is no restriction in the definition of $k_{a}$ and $k_{d}$, so that they may depend on geometrical considerations and on the local tracer density. Finally, the evolution of the propagated quantity associated with free tracers now includes a term accounting for the desorption of adsorbed particles:

$$
P(\mathbf{r}, t+\Delta t)=P^{\star}(\mathbf{r}, t+\Delta t)+P_{\mathrm{ads}}(\mathbf{r}, t) p_{d},
$$

where $P^{\star}$ is still given by Eq. (7). In order to compute the VACF of the tracers, one propagates as $P$ the probability to arrive at position $\mathbf{r}$ at time $t$, weighted by the initial velocity of tracers. Thus one needs to initialize, for each direction $\gamma$, a propagated quantity according to the Maxwell-Boltzmann 
distribution. The Boltzmann weights for solid $(\mathcal{S})$, fluid $(\mathcal{F})$, and interfacial $(\mathcal{I} \subset \mathcal{F})$ nodes read

$$
\begin{cases}0 & \text { for } \mathbf{r} \in \mathcal{S}, \\ e^{-\beta \mu^{e x}(\mathbf{r})} / \mathcal{Z} & \text { for } \mathbf{r} \in \mathcal{F} \backslash I, \\ e^{-\beta \mu^{e x}(\mathbf{r})}\left(1+e^{\left.-\beta \Delta \mu^{\mathrm{ads}(} \mathbf{r}\right)}\right) / \mathcal{Z} & \text { for } \mathbf{r} \in \mathcal{I},\end{cases}
$$

where $e^{-\beta \Delta \mu^{\text {ads }}(\mathbf{r})}=k_{a} /\left(k_{d} \Delta x\right)$ corresponds to the sorption free energy for interfacial tracers, $\beta \equiv 1 / k_{B} T$, with $k_{B}$ being the Boltzmann's constant and $T$ being the temperature, and $\mathcal{Z}$ is the partition function of the tracers. The excess chemical potential $\mu^{e x}(\mathbf{r})$ includes in the case of tracers with charge $q$ a mean-field electrostatic contribution $q \psi(\mathbf{r})$ with $\psi$ the local electrostatic potential. The VACF is then simply given as in the no sorption case [17] by

$$
Z_{\gamma}(t)=\sum_{\mathbf{r}} P(\mathbf{r}, t)\left(\sum_{i} p_{i}(\mathbf{r}, t) c_{i \gamma}\right) .
$$

\section{VALIDATION}

In order to validate this scheme, we compare numerical results and exact theoretical solutions of Eqs. (1) and (2). This allows us to assess the validity of our method independently of experimental results. We consider the diffusion and dispersion of tracers in a slit pore, i.e., between two walls at positions $x=0$ and $x=L$. The time-dependent diffusion coefficient $D(t)$ in the direction normal to the wall is given by [6]

$$
\begin{aligned}
\frac{D(t)}{D_{b}}= & \frac{1}{\left(2 k_{a}+k_{d} L\right)} \mathcal{L}^{-1}\left[\frac{k_{d} L}{\chi^{2}}\right. \\
& \left.-\frac{2 k_{d}\left(k_{d}+s\right) \sinh \kappa}{\chi^{3}\left[\left(k_{d}+s\right) \cosh \kappa+k_{a} \chi \sinh \kappa\right]}\right],
\end{aligned}
$$

where $s$ is the Laplace conjugate of time $t, \mathcal{L}^{-1}$ is the inverse Laplace transform, $\chi=\sqrt{s / D_{b}}$, and $\kappa=\chi L / 2$.

In Fig. 1, we compare the exact time-dependent diffusion coefficient $D(t)$ of Eq. (13) with the one extracted from

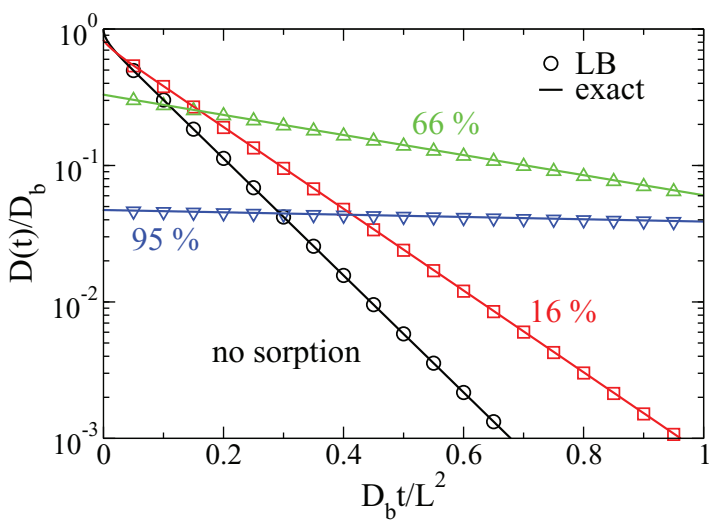

FIG. 1. (Color online) The time-dependent diffusion coefficient $D(t)$ normalized by the bulk diffusion coefficient $D_{b}$ of neutral tracers in a slit pore, as extracted from lattice Boltzmann simulations using our scheme (symbols) and from the reference exact solution of Eq. (13) (lines). Several fractions of adsorbed tracers, or sorption strength, $f_{a}$, defined in Eq. (14), are presented: black circles, 0\%; red squares, $16 \%$; green upward triangles, $66 \%$; and blue downward triangles, $95 \%$. lattice Boltzmann simulations for different sorption strength This last quantity is defined by the fraction of adsorbed tracers. Unless otherwise stated, all simulations are performed within a slit pore of width $L=100 \Delta x$ and a bulk diffusion coefficient $D_{b}=10^{-2} \Delta x^{2} / \Delta t$. These values are chosen very conservatively since the LB method is known to be efficient even in narrow slits (even for $L<10 \Delta x$ ) and for a wide range of magnitudes in the diffusion coefficients $[16,25]$. $L$ and $D_{b}$ therefore account for a negligible part of the difference with exact results. We can thus purposely assess the effect of the new algorithm only. In Fig. 1, we report timedependent diffusion coefficients calculated by our method for a fixed sorption rate $k_{a}=10^{-1} \Delta x / \Delta t$ and decreasing desorption rates $k_{d} \Delta t=10^{-2}, 10^{-3}$, and $10^{-4}$, resulting in an increasing fraction of adsorbed tracers $f_{a}$ of approximately $16 \%, 66 \%$, and $95 \%$, respectively. This fraction is
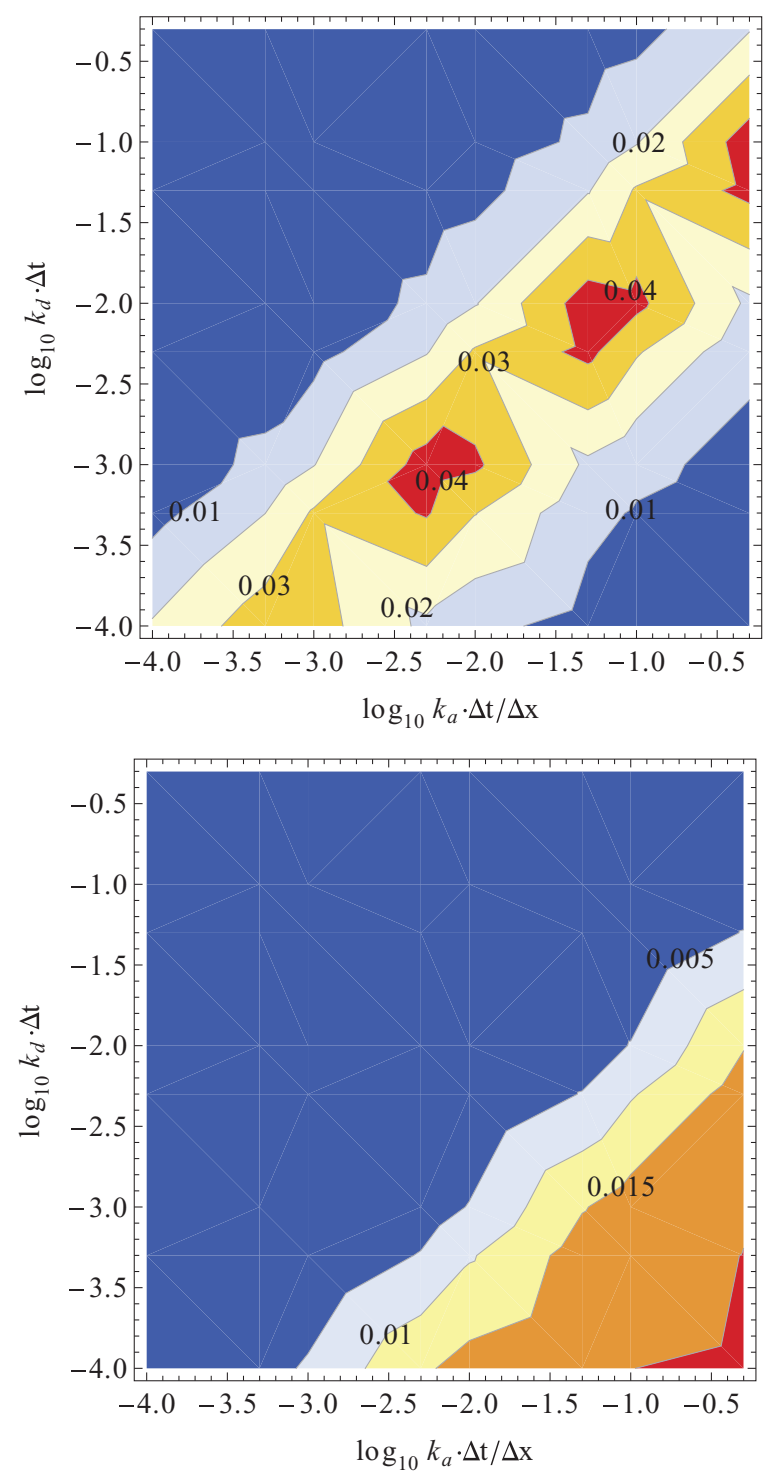

FIG. 2. (Color online) Contour plots of the relative error of our lattice Boltzmann scheme with respect to exact results, given by Eq. (13), on (top) the slope and (bottom) origin of the linear regression of the time-dependent diffusion coefficient of neutral tracers in a slit pore as a function of the adsorption and desorption rates $k_{a}$ and $k_{d}$. 
given by [6]

$$
f_{a}=\left(1+\frac{k_{d} L}{2 k_{a}}\right)^{-1}
$$

Excellent agreement is found between exact solutions and our numerical results for all fractions of adsorbed tracers, i.e., for all "sorption strengths."

At the initial time, $D(t=0)$ is given by the fraction of mobile tracers. At intermediate times, sorption and desorption processes significantly decrease the slope of $D(t)$, as the partial immobilization at the surface slows down the exploration of the pore. We have shown recently that for this range of parameters, this slope is given by $k_{d} /\left(1+k_{a} L / 2 D_{b}\right)$ [6]. In this illustration of diffusion between two walls, the confinement is total, so that for sufficiently long times, the effective diffusion coefficient tends to zero.

In order to quantify the error on $D(t)$ with respect to the exact result of Eq. (13), we plot in Fig. 2 the relative error on the slope and origin of the linear fit of $\log _{10} D(t)$ for long times, i.e., for $D_{b} t / L^{2}>0.5$, as a function of $k_{a}$ and $k_{d}$. In the whole range of $k_{a}$ and $k_{d}$, the relative errors on the slope and origin remain under $5 \%$ and $2 \%$, respectively, which is highly satisfactory.

The effect of a pressure gradient has also been studied on the same system. The resulting Poiseuille flow induces Taylor-Aris dispersion [32,33] of the tracers with a dispersion coefficient $K$, which is known exactly in the presence of adsorption and desorption in the simple slit geometry [5]:

$$
\frac{K}{D_{b}}=1+P_{e}^{2}\left[\frac{102 y^{2}+18 y+1}{210(1+2 y)^{3}}+\frac{D_{b}}{L^{2} k_{d}} \frac{2 y}{(1+2 y)^{3}}\right]
$$

where $P_{e}=L \bar{v} / D_{b}$ is the Péclet number and $y=k_{a} / k_{d} L$.

In Fig. 3, we compare the dispersion coefficient as calculated by LB with the exact results of Eq. (15) for various sorption strengths as a function of the Péclet number. Adsorption significantly increases the dispersion, as it slows down part of the tracers. The agreement between our scheme

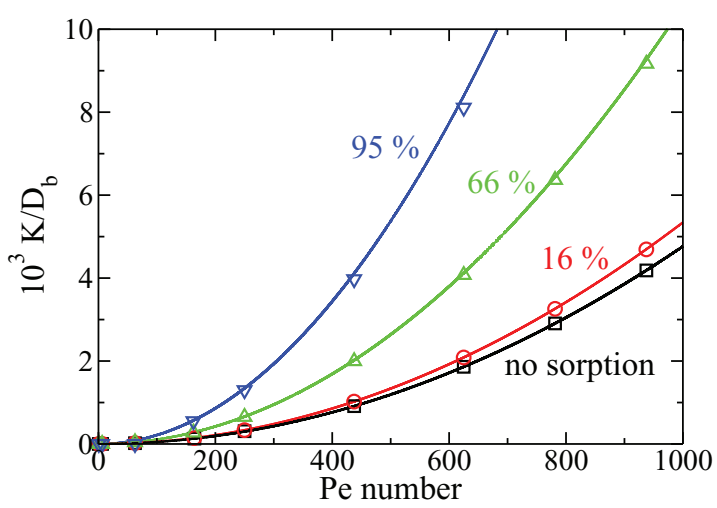

FIG. 3. (Color online) Dispersion coefficient of neutral tracers in a slit pore in the direction of the flux, normalized by the bulk diffusion coefficient, as a function of the Péclet number, as extracted from our lattice Boltzmann scheme (symbols) and from the exact results (lines). Several fractions of adsorbed tracers, or sorption strength, $f_{a}$, defined in Eq. (14), are presented: black squares, $0 \%$; red circles, $16 \%$; green upward triangles, $66 \%$; and blue downward triangles, $95 \%$.

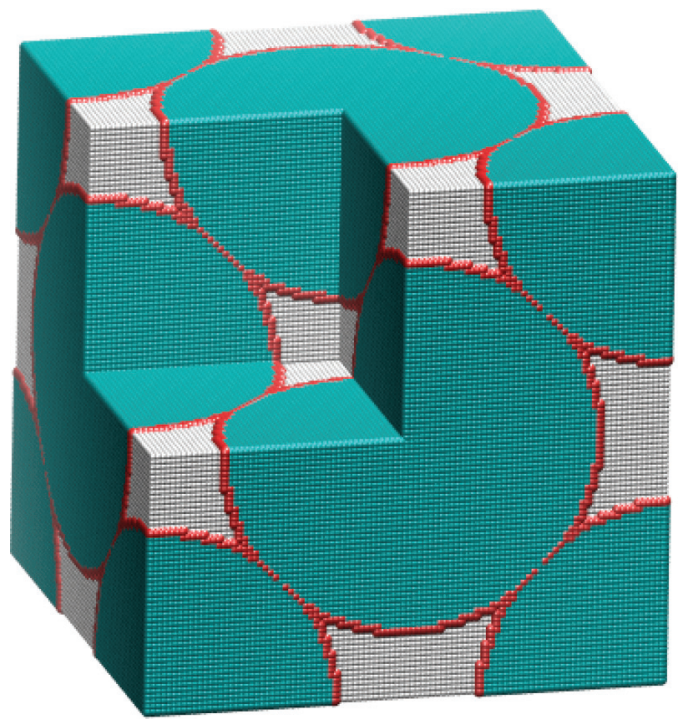

FIG. 4. (Color online) Perspective view of a unit cell of a fcc packing of spheres of lattice parameter $100 \Delta x$. Solid nodes are colored in blue. Interfacial fluid nodes, where adsorption processes may occur, are colored in red. Noninterfacial fluid nodes are in white.

and the exact results is excellent, even for strong adsorption and Péclet numbers above 100.

As mentioned above, the time dependence of the diffusion coefficient is a signature of the intrinsic geometric properties of a porous medium. The simplest model of such media consists of a compact fcc lattice of spheres of radius $R$ [34]. The porosity, i.e., the fraction of empty (or fluid) space, is $1-\pi /(3 \sqrt{2}) \approx 26 \%$. The unit cell contains four octahedral cavities of radius $\approx 0.41 R$ connected by eight smaller tetrahedral cavities of radius $\approx 0.22 R$ by a small channels of radius $\approx 0.15 R$. This fcc lattice is illustrated in Fig. 4 for a lattice parameter $L=100 \Delta x$.

We report the time-dependent diffusion coefficient for this model porous medium in Fig. 5. At $t=0$, the diffusion

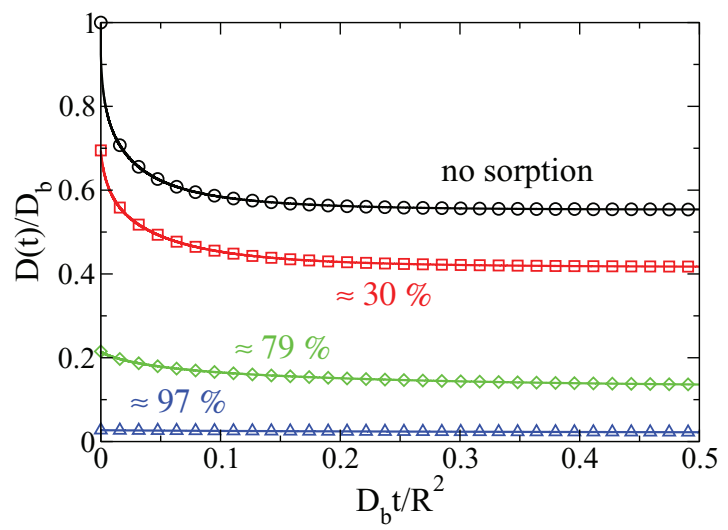

FIG. 5. (Color online) Diffusion coefficient $D(t)$ of a neutral tracer, normalized by the bulk diffusion coefficient $D_{b}$ in the fcc packing of spheres with radius $R$ illustrated in Fig. 4, as a function of the reduced time. Several fractions of adsorbed tracers, or sorption strength, $f_{a}$, defined in Eq. (14), are presented: black circles, $0 \%$, i.e., without adsorption; red squares, $30 \%$; green diamonds, $79 \%$; and blue triangles, $97 \%$. 
coefficient is again given by the fraction of free particles times their bulk diffusion coefficient. After a reduced time $D_{b} t / R^{2}=0.5$, tracers have explored the whole porosity, and the diffusion coefficient tends toward the effective diffusion coefficient. The time dependence is strongly influenced by adsorption and desorption, as in the slit pore case. It is thus essential to consider these phenomena when interpreting experimental measurements of effective and time-dependent diffusion coefficients.

\section{CONCLUSION}

In summary, we have proposed a scheme that accounts for adsorption and desorption in a generic lattice Boltzmann scheme, allowing for the calculation of mesoscale dynamical properties of tracers in media of arbitrary complexity. These processes are modeled by kinetic rates of adsorption and desorption taking place at interfacial, fluid nodes. The algorithm has been validated over a wide range of adsorption and desorption rates and Péclet numbers in the slit pore geometry where exact results are available. Finally, we have shown on a more complex porous medium that adsorption and desorption processes may not be neglected, as they strongly modify the short, intermediate, and long time behaviors of the diffusion coefficient as well as the dispersion coefficient. In turn, this demonstrates that neglecting interactions with the surface in the interpretation of $D(t)$ as a probe of the geometry of the porous medium, as measured experimentally, e.g., by PSGE-NMR, may lead to incorrect conclusions. This scheme may now be used in two ways. First, one could predict the effective diffusion coefficient in complex heterogeneous media for species with known adsorption and desorption rates (from experiments or molecular simulations). Conversely, from reference measurements of the time-dependent diffusion coefficient in controlled geometries, one could extract the adsorption and desorption rates $k_{a}$ and $k_{d}$. Moreover, in the case of diffusion in the solid stationary phase, the method would allow us to relate the relevant diffusion constant to the shape of the elution profile.

While the present method is very general and also applies in principle to the case of irreversible adsorption $\left[k_{d}=0\right.$, i.e., $p_{d}=0$ in Eq. (10)], such a situation is of interest only outside of equilibrium. Indeed, in that case at equilibrium all the solute is adsorbed on the surface, and its VACF corresponds to the sorbed species only. The propagation scheme [Eqs. (7)(10)] could nevertheless be used to investigate irreversible adsorption out of equilibrium by considering the density as the propagated quantity $P$ (instead of the one described here to compute the VACF), as was done, e.g., by Warren to simulate electrokinetic phenomena [35]. As an example of practical application where (possibly irreversible) sorption is coupled to electrokinetic phenomena, we can, for example, mention the case of ion adsorption onto charged minerals such as clays.

\section{ACKNOWLEDGMENTS}

B.R. and M.L. acknowledge financial support from the French Agence Nationale de la Recherche under Grant No. ANR-09-SYSC-012. M.D. acknowledges the French Agence Nationale pour la Gestion des Déchets Radioactifs (ANDRA) for financial support. I.P. acknowledges Spanish MINECO (Project No. FIS2011-22603) and DURSI (SGR2009-634) for financial support. D.F. acknowledges a Wolfson Merit Award from the Royal Society of London.
[1] P. P. Mitra, Phys. A 241, 122 (1997).

[2] P. N. Sen, J. Chem. Phys. 119, 9871 (2003).

[3] P. N. Sen, Concepts Magn. Reson. A 23, 1 (2004).

[4] O. K. Dudko, A. M. Berezhkovskii, and G. H. Weiss, J. Phys. Chem. B 109, 21296 (2005).

[5] M. Levesque, O. Bénichou, R. Voituriez, and B. Rotenberg, Phys. Rev. E 86, 036316 (2012).

[6] M. Levesque, O. Bénichou, and B. Rotenberg, J. Chem. Phys. 138, 034107 (2013).

[7] U. Tallarek, D. van Dusschoten, H. Van As, E. Bayer, and G. Guiochon, J. Phys. Chem. B 102, 3486 (1998).

[8] D. Alcor, V. Croquette, L. Jullien, A. Lemarchand, and H. L. Swinney, Proc. Natl. Acad. Sci. USA 101, 8276 (2004).

[9] A. Gunnarsson, P. Jonsson, R. Marie, J. O. Tegenfeldt, and F. Hook, Nano Lett. 8, 183 (2007).

[10] R. Verberg and A. J. C. Ladd, Phys. Rev. E 65, 056311 (2002).

[11] C. Lowe and D. Frenkel, Phys. A 220, 251 (1995).

[12] C. P. Lowe and D. Frenkel, Phys. Rev. Lett. 77, 4552 (1996).

[13] F. Capuani, D. Frenkel, and C. P. Lowe, Phys. Rev. E 67, 056306 (2003).

[14] B. Rotenberg, I. Pagonabarraga, and D. Frenkel, Faraday Discuss. 144, 223 (2010).
[15] M. A. van der Hoef and D. Frenkel, Phys. Rev. A 41, 4277 (1990).

[16] R. Merks, A. Hoekstra, and P. Sloot, J. Comput. Phys. 183, 563 (2002).

[17] B. Rotenberg, I. Pagonabarraga, and D. Frenkel, Europhys. Lett. 83, 34004 (2008).

[18] I. Pagonabarraga, B. Rotenberg, and D. Frenkel, Phys. Chem. Chem. Phys. 12, 9566 (2010).

[19] M. Wang and Q. Kang, Anal. Chem. 81, 2953 (2009).

[20] M. Wang and Q. Kang, J. Comput. Phys. 229, 728 (2010).

[21] M. Wang, Q. Kang, H. Viswanathan, and B. A. Robinson, J. Geophys. Res. 115, B10205 (2010).

[22] U. Frisch, B. Hasslacher, and Y. Pomeau, Phys. Rev. Lett. 56, 1505 (1986)

[23] G. R. McNamara and G. Zanetti, Phys. Rev. Lett. 61, 2332 (1988).

[24] A. J. C. Ladd, J. Fluid Mech. 271, 285 (1994).

[25] A. J. C. Ladd, J. Fluid Mech. 271, 311 (1994).

[26] S. Succi, The Lattice Boltzmann Equation: For Fluid Dynamics and Beyond (Oxford University Press, Oxford, 2001).

[27] C. K. Aidun and J. R. Clausen, Annu. Rev. Fluid Mech. 42, 439 (2010).

[28] S. Chen and G. D. Doolen, Annu. Rev. Fluid Mech. 30, 329 (1998). 
[29] R. S. Maier, D. M. Kroll, H. T. Davis, and R. S. Bernard, Int. J. Mod. Phys. C 9, 1523 (1998).

[30] E. S. Boek and M. Venturoli, Comput. Math. Appl. 59, 2305 (2010).

[31] C. Lowe, D. Frenkel, and M. Hoef, J. Stat. Phys. 87, 1229 (1997).
[32] G. Taylor, Proc. R. Soc. London, Ser. A 219, 186 (1953).

[33] R. Aris, Proc. R. Soc. London, Ser. A 235, 67 (1956).

[34] R. J. Hill, D. L. Koch, and A. J. C. Ladd, J. Fluid. Mech. 448, 213 (2001).

[35] P. B. Warren, Int. J. Mod. Phys. C 8, 889 (1997). 